\title{
Efficacy of a physiotherapy rehabilitation program for individuals undergoing arthroscopic management of femoroacetabular impingement - the FAIR trial: a randomised controlled trial protocol
}

Kim L Bennell ${ }^{*}$, John M O'Donnell ${ }^{2}$, Amir Takla ${ }^{3}$, Libby N Spiers ${ }^{1}$, David J Hunter ${ }^{4}$, Margaret Staples ${ }^{5}$ and Rana S Hinman ${ }^{1}$

\begin{abstract}
Background: Femoroacetabular impingement is a common cause of hip/groin symptoms and impaired functional performance in younger sporting populations and results from morphological abnormalities of the hip in which the proximal femur abuts against the acetabular rim. Many people with symptomatic femoroacetabular impingement undergo arthroscopic hip surgery to correct the bony abnormalities. While many case series over the past decade have reported favourable surgical outcomes, it is not known whether formal rehabilitation is needed as part of the management of patients undergoing this surgical procedure. This randomised controlled trial will investigate the efficacy of a progressive physiotherapist-supervised rehabilitation program (Takla-O'Donnell Protocol) in improving health-related quality of life, physical function and symptoms in individuals undergoing arthroscopic management of femoroacetabular impingement.
\end{abstract}

Methods/design: 100 people aged 16-35 years undergoing hip arthroscopy for symptomatic femoroacetabular impingement will be recruited from surgical practices in Melbourne, Australia and randomly allocated to either a physiotherapy or control group. Both groups will receive written information and one standardised post-operative physiotherapy visit whilst in hospital as per usual care. Those in the physiotherapy group will also receive seven individual 30-minute physiotherapy sessions, including one pre-operative visit (within 2 weeks of surgery) and six post-operative visits at fortnightly intervals (commencing two weeks after surgery). The physiotherapy intervention will incorporate education and advice, manual techniques and prescription of a progressive rehabilitation program including home, aquatic and gym exercises. The control group will not receive additional physiotherapy management. Measurements will be taken at baseline (2 weeks pre-operatively) and at 14 and 24 weeks post-surgery. Primary outcomes are the International Hip Outcome Tool and the sports subscale of the Hip Outcome Score at 14 weeks post-surgery. Secondary outcomes include the Copenhagen Hip and Groin Outcome Score, the activities of daily living subscale of the Hip Outcome Score, the Heidelberg Sports Activity Score, a modified Tegner Activity Scale and participant-perceived overall change.

(Continued on next page)

\footnotetext{
* Correspondence: k.bennell@unimelb.edu.au

'The University of Melbourne, Centre for Health, Exercise and Sports Medicine, Department of Physiotherapy, School of Health Sciences, Melbourne, Vic, Australia

Full list of author information is available at the end of the article
} 


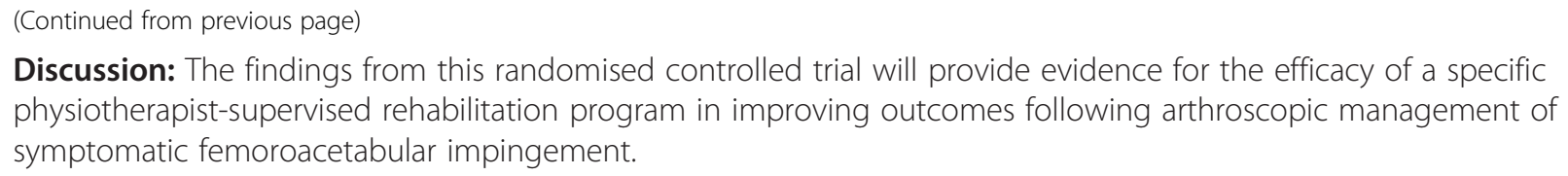

Trial registration: Australian New Zealand Clinical Trials Registry reference number: ACTRN12613000282785.

Keywords: Physiotherapy, Physical therapy, Rehabilitation, Hip arthroscopy, Femoroacetabular impingement

\section{Background}

Femoroacetabular impingement (FAI) is a common cause of hip/groin symptoms and impaired performance in younger sporting populations [1-3]. FAI is the result of morphological abnormalities of the hip in which the proximal femur abuts against the acetabular rim. This impingement is due either to abnormalities in the morphology of the femoral head (referred to as cam impingement) and/or excessive acetabular coverage of the femoral head (referred to as pincer impingement) [4]. Not only can FAI give rise to symptoms and impair function, the repetitive inappropriate bony contact can also lead to a cascade of structural damage including tearing at the chondrolabral junction, full thickness cartilage delamination and eventually hip osteoarthritis [5,6].

Hip arthroscopy is often used in the assessment and management of hip pathology. Indeed the total number of hip arthroscopies performed around the world is rapidly increasing [7]. Currently, post-operative management of patients following hip arthroscopy for FAI is variable, and dependent on surgeon's preferences and patient access to rehabilitation services. Some patients undergo formal physiotherapist-supervised rehabilitation, whilst others do not. Physiotherapist-supervised rehabilitation is advocated in the literature in order to restore muscle function and strength and improve joint range of motion, as well as facilitate a safe and graded return to sporting activity. Several rehabilitation protocols following hip arthroscopy have been described in the literature [8-14]. These impose varying post-operative restrictions related to weightbearing and hip range of motion, and utilise different exercise and therapeutic techniques; however there is no high quality evidence that any one is more effective than another, or indeed, more effective than no formal postoperative rehabilitation.

Due to the dearth of research in this area, evidencebased recommendations to guide the post-operative management of patients following hip arthroscopy for FAI cannot be made. Accordingly, based on their extensive clinical experience, two of our team members (AT physiotherapist and JOD orthopaedic surgeon) developed a specific physiotherapist rehabilitation program (Takla-O'Donnell Protocol (TOP)), designed to facilitate return to sport typically within three months of surgery. Whilst anecdotally this protocol appears effective, it has not been subjected to formal research evaluation. Thus, the objective of this randomised controlled trial (RCT) is to evaluate the efficacy of the TOP, a progressive physiotherapist-supervised rehabilitation intervention, on health-related quality of life, physical function and symptoms in individuals undergoing hip arthroscopic management of FAI. The primary time point will be measured at 14 weeks post-surgery, a time when typically patients would have completed the TOP rehabilitation and returned to their usual activities.

Our primary hypothesis is that in individuals undergoing hip arthroscopy for symptomatic FAI, those in the physiotherapy group (PT) will report significantly greater improvements in health-related quality of life, as measured by the International Hip Outcome Tool (iHOT-33), and function in sport, as measured by the sports subscale of the Hip Outcome Score (HOS), at 14 weeks post surgery than those in the control group $(\mathrm{CON})$ not undergoing formal rehabilitation.

\section{Methods/design \\ Trial design}

We will conduct a parallel-design 2-arm RCT with outcomes assessed at baseline (within 2 weeks prior to surgery), at 14 weeks post surgery (immediately following the PT intervention) and at 24 weeks post-surgery, with the primary outcome time point being 14 weeks post surgery. Reporting of the study will conform to CONSORT guidelines for non-pharmacological studies [15].

\section{Participants}

100 men and women aged 16-35 years with symptomatic FAI who are scheduled for hip arthroscopy will be recruited from the surgical practices of five orthopaedic surgeons in metropolitan Melbourne, Victoria, Australia.

People will be eligible if they have (i) had hip/groin symptoms for at least 3 months; (ii) been diagnosed with FAI by an orthopaedic surgeon based on symptoms, clinical signs and imaging findings; and (iii) are scheduled for hip arthroscopy.

People will be excluded from participating if they (i) have radiographic evidence of hip osteoarthritis that is more than mild in severity defined as Tonnis $>$ grade 1 [16]; (ii) are a professional athlete; (iii) have other concurrent injury/conditions that will affect their ability to 
participate in the rehabilitation program and/or assessment procedures; (iv) are unable to attend a study physiotherapist or participate in the rehabilitation program if randomised to the PT group; (v) wish to undertake formal supervised rehabilitation following hip arthroscopy; and (vi) are unable to understand English.

Ethical approval has been obtained from the University of Melbourne Human Research Ethics Committee (HREC No. 1238190). All participants will provide written informed consent.

\section{Study procedure}

Patients who are scheduled for hip arthroscopic surgery and fulfil the eligibility criteria will be identified by the surgeon and provided with study information by staff at the surgeon's practice. An independent research assistant will confirm eligibility via subsequent telephone screening. Consenting participants will complete baseline questionnaires electronically or via post approximately two weeks prior to surgery. Upon receipt of baseline data, participants will be consecutively randomised into either the PT or the CON group by an independent person not involved in recruitment, assessment or treatment of participants. Participants in both groups will undergo hip arthroscopy for management of their FAI as scheduled by their surgeon and will receive standardised pre- and post-operative care, including an in-patient physiotherapy visit, provision of written educational material and a follow-up appointment with the orthopaedic surgeon at approximately two weeks post surgery. The PT group will additionally receive seven individual 30-minute physiotherapy sessions including one pre-operative session and six post-operative sessions at fortnightly intervals commencing two weeks after surgery. The PT group will also perform a home, aquatic and gym rehabilitation program. Re-assessment will occur at 14 weeks (following completion of physiotherapy rehabilitation in the PT group) and 24 weeks post surgery via administration of questionnaires completed online or via post. A flow chart outlining the study procedures is shown in Figure 1.

\section{Blinding}

It is not possible to blind participants in this study. The study physiotherapists treating participants in the PT group will be, by necessity, unblinded. The researcher managing the patient-completed data will be blinded to group allocation as will the physiotherapists providing in-patient treatment to both groups. The orthopaedic surgeon will also be blinded to group allocation and participants will be asked not to disclose this to the surgeon at their follow-up appointment. The statistician will be blinded to group allocation until completion of the statistical analyses.

\section{Randomisation and allocation concealment}

The randomisation schedule will be prepared by the study biostatistician using a computer-generated random numbers table. There will be a 1:1 allocation ratio of participants to the PT and CON groups. Randomisation will be conducted by random permuted blocks of varying size, and stratified by orthopaedic surgeon (so that each surgeon contributes approximately equal numbers in each group to control for surgical variation) as well as by whether the participant is having unilateral or bilateral surgery. Participants in the PT group will choose their preferred project physiotherapist according to geographical convenience.

Consecutively numbered, sealed, opaque envelopes containing group allocation will be prepared by a researcher with no other involvement in the study. The envelopes will be stored in a locked location and will be opened in sequence to reveal group allocation by a researcher not involved in recruitment, treatment or assessment of outcomes.

\section{Interventions}

All participants will receive hip arthroscopic surgery for FAI performed by an experienced hip orthopaedic surgeon. Unstable articular cartilage flaps will be debrided (chondroplasty) and any exposed subchondral bone will be treated by microfracture if its area is $<400 \mathrm{sq} \mathrm{mm}$. Superficial labral tears will be debrided and tears that are unstable or $>50 \%$ deep will be repaired. No segmental labral resections will be performed. Partial or complete tears of the ligamentum teres will be debrided with a radiofrequency probe. Cam impingement lesions will be treated by femoral osteochondroplasty. Pincer impingement lesions will be treated when there is both radiological and intra-operative pathological evidence to confirm the diagnosis. At the end of the procedure the operative field will be injected with $5 \mathrm{mgs}$ Morphine, $30 \mathrm{mgs}$ Ketorolac and $20 \mathrm{ml}$ of $0.2 \%$ Naropin.

Immediate postoperative care at St Vincent's Private Hospital East Melbourne and Bellbird Private Hospital will be consistent for both groups. Patients will stay in hospital overnight, unless they specifically request to leave on the same day. Ice and compression will be applied to the hip overnight. Standard care will include (i) an in-patient physiotherapy visit for provision of a gait aid; (ii) the surgeons' usual inpatient written educational material covering post-operative precautions, return to activity and basic hip exercises; and (iii) use of a nonsteroidal anti-inflammatory drug, as selected by the surgeon, for one month as prophylaxis against heterotopic bone formation in those without contraindications to their use. A follow-up visit with the orthopaedic surgeon will occur at approximately two weeks post surgery. 


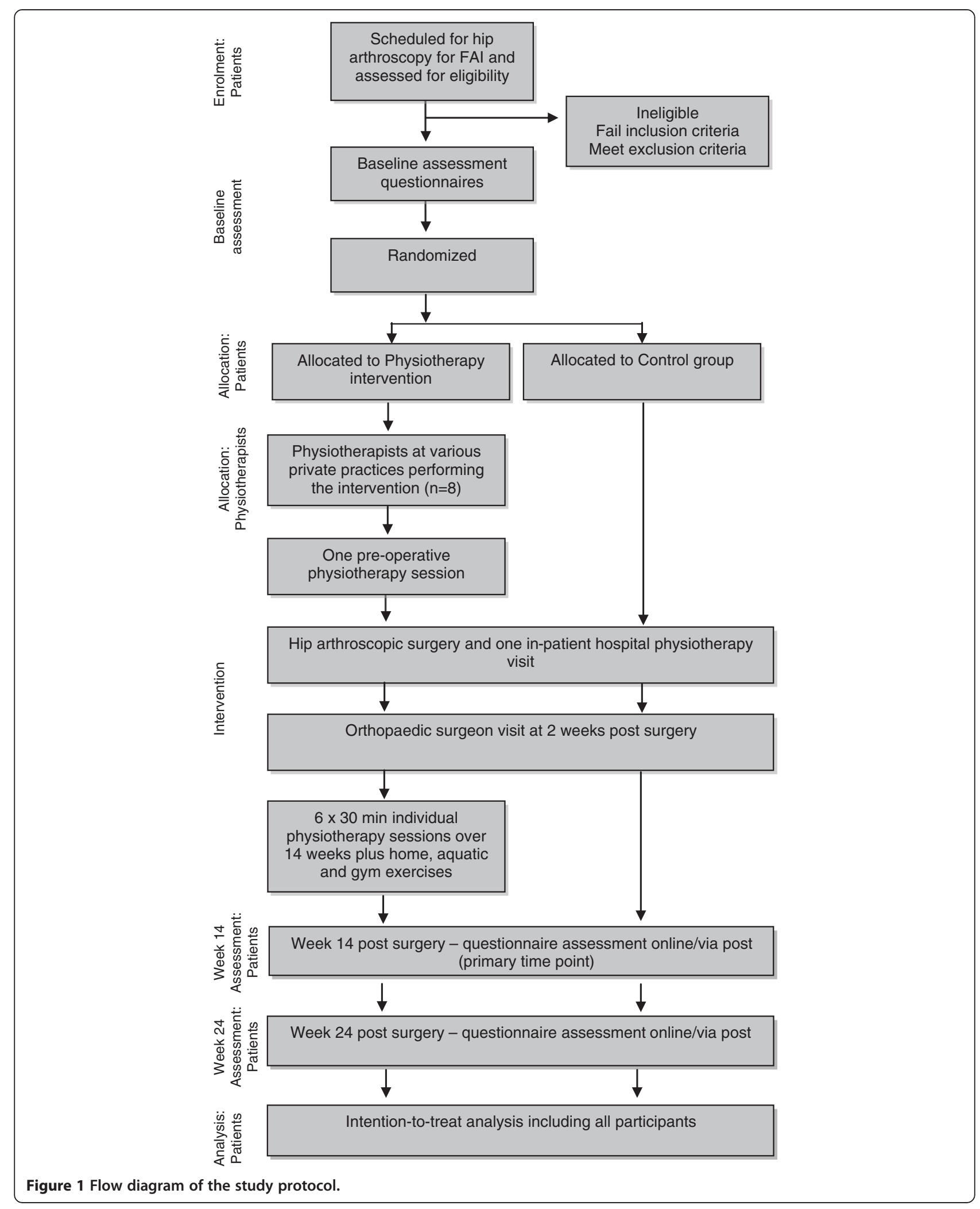


Patients will be asked to use crutches until they can walk without pain and without limping, likely 10 days or less. Patients will be advised to avoid hip flexion past 90 degrees for approximately six weeks and to avoid positions that have the potential to cause impingement and increase inflammation. This will include avoidance of deep squatting, lifting heavy objects from the floor, pivoting or twisting on a fixed foot, sitting with the hip flexed past 90 degrees, as well as prolonged sitting.

\section{Physiotherapy}

Participants in the PT group will attend a study physiotherapist for one pre-operative visit (after baseline assessment) within two weeks prior to surgery, and six post-operative visits on a fortnightly basis commencing at week two (approximately 2, 4, 6, 8, 10 and 12 weeks post surgery). Each session will be a 30 -minute individual appointment. Physiotherapists who have at least two years of musculoskeletal experience and work in private clinics in metropolitan Melbourne and regional Victoria will be trained to provide the physiotherapy intervention.

The physiotherapists will follow a progressive semistructured program based on the Takla-O'Donnell Protocol, a clinical protocol developed and refined by two of the authors over a 10-year period. It will comprise of standardised assessments/re-assessments, education and advice, manual therapy techniques, prescription and progression of a home, aquatic and gym program, and graduated return to sport and physical activity. A summary of the physiotherapy intervention is provided in Tables 1, 2, 3, 4. Participants will receive handouts demonstrating the home exercises as well as a log-book to record completion of home, aquatic and gym sessions.

Education Education and advice will be a focus of the preoperative treatment session as well as an important aspect of the first post-operative session. This will include information regarding post-operative joint protection (such as activity avoidance or modification), return to driving and work, and the importance of the home exercise program.

Manual therapy Manual therapy techniques will be used throughout the rehabilitation program. Trigger point massage will be used at each post-surgical treatment session to release muscle tension, assist with pain relief and improve hip range of motion [17]. Lumbar spine mobilisation, in the form of passive accessory intervertebral movements, will be performed [18] in those patients where the physiotherapy assessment determines it is required.

Deep hip rotator muscle strengthening A key component of the home program is local stabilization of the hip joint by retraining and strengthening the deep hip rotator muscles. This deep musculature includes quadratus femoris, the gemelli, and obturator internus. These muscles have a short lever arm and therefore have the potential to act as deep stabilizers, to steady the femoral head in the acetabulum. It has been suggested that they may provide fine control of hip joint stability, acting as the "rotator cuff" of the hip joint $[19,20]$. There is some evidence that these deep muscles contribute to dynamic hip stability $[21,22]$ and therefore it is possible that retraining and strengthening of this group may accelerate rehabilitation post hip arthroscopy.

Deep hip rotator muscle retraining follows seven stages, with the participant moving to the next stage once they achieve effective activation and endurance of the deep hip rotators required at that particular stage as determined by the therapist. Exercise sheets provided to study participants show these stages in more detail [see Additional file 1]. Retraining commences pre-operatively in prone, followed by progression to 4-point-kneeling, the addition of resistance band and finally weight-bearing with visual feedback and global muscle recruitment.

Table 1 The physiotherapy intervention - manual therapy techniques

\begin{tabular}{|c|c|c|c|c|}
\hline Manual Therapy Techniques & Aim & Description & Timeframes & Dosage \\
\hline \multicolumn{5}{|l|}{ Mandatory technique: } \\
\hline $\begin{array}{l}\text { Trigger point massage of rectus } \\
\text { femoris, adductors, tensor fascia latae/ } \\
\text { gluteus medius/gluteus minimus and } \\
\text { pectineus muscles and associated fascia }\end{array}$ & $\begin{array}{l}\text { To address soft tissue } \\
\text { restrictions with the aim of } \\
\text { reducing pain and } \\
\text { improving hip range of } \\
\text { movement }\end{array}$ & $\begin{array}{l}\text { Sustained pressure trigger point } \\
\text { release with the muscle on stretch. In } \\
\text { general, mobilise restrictions laterally } \\
\text { to the line of tension of the muscle } \\
\text { being treated }\end{array}$ & Session 2-7 & $\begin{array}{l}30-60 \text { seconds } \\
\text { per trigger point }\end{array}$ \\
\hline \multicolumn{5}{|l|}{ Optional technique: } \\
\hline $\begin{array}{l}\text { Lumbar spine mobilisation, if indicated } \\
\text { by lumbar spine physiotherapy } \\
\text { assessment }\end{array}$ & $\begin{array}{l}\text { To improve mobility and } \\
\text { pain-free movement of the } \\
\text { lumbar spine to assist with } \\
\text { hip function }\end{array}$ & $\begin{array}{l}\text { Unilateral postero-anterior accessory } \\
\text { glides, Grade III or IV }\end{array}$ & Session 3-12 & $\begin{array}{l}3-5 \text { sets of } 30- \\
60 \text { seconds }\end{array}$ \\
\hline
\end{tabular}

The treatment program is semi-structured, and includes a number of mandatory components plus some optional components. Individual program progression will be guided by assessment findings and the nature of the surgical procedure. 
Table 2 Home exercises

\begin{tabular}{|c|c|c|c|c|}
\hline Home exercises & Aim & Description & Timeframes & Dosage \\
\hline $\begin{array}{l}\text { Deep hip rotator muscle } \\
\text { retraining (see Additional file 1) }\end{array}$ & $\begin{array}{l}\text { Optimise hip } \\
\text { neuromuscular control } \\
\text { and improve dynamic } \\
\text { stability of the hip }\end{array}$ & $\begin{array}{l}\text { Seven stages progressing through prone, four- } \\
\text { point-kneel and dynamic standing positions, } \\
\text { with and without additional resistance. }\end{array}$ & Pre-op to session 7 & $\begin{array}{l}1 \text { minute, } 3-6 \\
\text { times per day }\end{array}$ \\
\hline Anterior hip stretch & $\begin{array}{l}\text { Assist in regaining full hip } \\
\text { extension range of } \\
\text { movement }\end{array}$ & $\begin{array}{l}\text { Supine in modified Thomas Test position with } \\
\text { the affected leg over the side of the bed. The } \\
\text { hip is extended until a stretch is felt at the front } \\
\text { of the hip }\end{array}$ & Session $2-4$ & 5 minutes daily \\
\hline $\begin{array}{l}\text { Hip flexion/extension in four- } \\
\text { point kneel - "pendulum" } \\
\text { exercise }\end{array}$ & $\begin{array}{l}\text { Prevent adhesions, } \\
\text { especially in those with } \\
\text { labral repair }\end{array}$ & $\begin{array}{l}\text { Four point kneel with gentle pendular swing of } \\
\text { the affected leg into hip flexion and extension } \\
\text { as far as comfortable }\end{array}$ & Session 2- 5 & 1 minute daily \\
\hline Posterior capsule stretch & & $\begin{array}{l}\text { Lying on unaffected side with the affected hip } \\
\text { as close to } 90 \text { degrees flexion as comfortable } \\
\text { and affected leg over the side of the bed. }\end{array}$ & $\begin{array}{l}\text { Session } 3-7 \text { (or } \\
\text { session } 4-7 \text { if } \\
\text { microfracture present) }\end{array}$ & $3 \times 30$ seconds \\
\hline
\end{tabular}

Stretches and range of motion exercises Pendular exercise (hip flexion/extension) in 4-point-kneel is included from two to ten weeks post-surgery, to reduce the risk of adhesions. Anterior hip joint capsule stretch (supine with the leg over the edge of a bed) is carried out daily from two to six weeks post-surgery to maximise hip extension range of movement. Posterior hip joint capsule stretch in side-lying commences four weeks after surgery (six weeks if microfracture present) and continues until hip external rotation range is equal to the other side. Exercise sheets provided to study participants in the PT group show these in more detail [see Additional file 1].

Gym and aquatic program A gym and aquatic program will commence two weeks post-surgery, following the first post-operative physiotherapy session. This will initially consist of walking in the pool and use of a stationary bike and cross-trainer, with progression to swimming and then resistance exercise for the lower body. Participants will be provided with access to local community gym and pool facilities (YMCA centres) and will be asked to carry out this aspect of the program at least twice weekly.

Return to sport The physiotherapists will provide guidance to participants regarding graduated return to sport. This will include provision of functional and sport-specific drills. Therapists will be guided by a table of options arranged to suggest drills appropriate for different sporting activities. This table is provided in Additional file 2. Generally, preliminary components of sporting activity will begin six to eight weeks after surgery, and training in the actual sporting environment will commence 10 to 12 weeks after surgery.

\section{Control}

Participants in this group will not attend a study physiotherapist and will be requested to not undertake a formal

Table 3 Gym/aquatic program

\begin{tabular}{|c|c|c|c|c|}
\hline $\begin{array}{l}\text { Gym/aquatic } \\
\text { program }\end{array}$ & Aim & Description & Timeframes & Frequency \\
\hline Stationary cycling & $\begin{array}{l}\text { To improve hip range } \\
\text { of motion }\end{array}$ & $\begin{array}{l}\text { Upright bike with high seat to avoid } \\
\text { hip flexion past } 90 \text { degrees. Initially } 15 \text { mins } \\
\text { at moderate intensity }\end{array}$ & $\begin{array}{l}\text { Session } 2 \text { onwards } \\
\text { (Session } 3 \text { if have microfracture) }\end{array}$ & $2 \times$ weekly \\
\hline Walking in pool & $\begin{array}{l}\text { To maintain cardiovascular } \\
\text { fitness and improve hip range } \\
\text { of motion }\end{array}$ & $\begin{array}{l}\text { Walking at chest depth, forwards, } \\
\text { straight lines only. } 10 \text { mins for FOC or labral repair, } \\
5 \text { mins for microfracture or ligamentum teres repair }\end{array}$ & $\begin{array}{l}\text { Session } 2 \text { onwards } \\
\text { (session } 3 \text { if have microfracture) }\end{array}$ & $2 \times$ weekly \\
\hline Swimming & $\begin{array}{l}\text { To maintain/regain } \\
\text { cardiovascular fitness }\end{array}$ & $\begin{array}{l}\text { No kicking until 6-8 weeks post-surgery, } \\
500 \mathrm{~m}-1 \mathrm{~km}\end{array}$ & $\begin{array}{l}\text { Session } 2 \text { onwards } \\
\text { (session } 3 \text { if have microfracture) }\end{array}$ & $2 \times$ weekly \\
\hline Cross trainer & $\begin{array}{l}\text { To maintain/regain } \\
\text { cardiovascular fitness }\end{array}$ & Initially 15 mins at moderate intensity & $\begin{array}{l}\text { Session } 2 \text { onwards } \\
\text { (Session } 3 \text { if have microfracture) }\end{array}$ & $2 \times$ weekly \\
\hline $\begin{array}{l}\text { Squats } \\
\text { Lunges }\end{array}$ & $\begin{array}{l}\text { To improve lower limb } \\
\text { strength and function }\end{array}$ & $\begin{array}{l}3 \text { sets of } 10 \text { repetitions, working at } \\
\text { "moderately hard" on modified Rating } \\
\text { of Perceived Exertion (RPE) }\end{array}$ & Session 6 onwards & $2 \times$ weekly \\
\hline \multicolumn{5}{|l|}{ Leg press } \\
\hline \multicolumn{5}{|l|}{ Leg extensions } \\
\hline Hamstring curls & & & & \\
\hline
\end{tabular}


Table 4 Functional program

\begin{tabular}{|c|c|c|c|}
\hline $\begin{array}{l}\text { Functional } \\
\text { program }\end{array}$ & Description & Timeframes & Dosage \\
\hline Jogging & $\begin{array}{l}\text { Jogging on running track or grass, with affected leg to } \\
\text { the outside of the track ie anticlockwise for the right } \\
\text { hip. One lap of oval should be approx } 400 \mathrm{~m} \text {. }\end{array}$ & $\begin{array}{l}\text { Session } 4 \text { onwards for FOC } \\
\text { (femoral osteochrondroplasty) } \\
\text { only, session } 5 \text { onwards for others }\end{array}$ & $\begin{array}{l}3 \times \text { weekly } 6 \text { laps in first week, } 8 \text { laps } \\
\text { in second week, } 10 \text { laps in third week } \\
\text { (ie building up to } 4 \mathrm{~km} \text { ) }\end{array}$ \\
\hline \multirow{2}{*}{$\begin{array}{l}\text { Acceleration/ } \\
\text { change of } \\
\text { direction drills }\end{array}$} & \multirow[t]{2}{*}{ Zig-zag jogging } & Session 5 (FOC only) & \multirow{2}{*}{$\begin{array}{l}\text { Dependent on sport goals and } \\
\text { surgical procedure }\end{array}$} \\
\hline & & Session 6 all others & \\
\hline \multirow{2}{*}{$\begin{array}{l}\text { Sport-specific } \\
\text { drills }\end{array}$} & \multirow{2}{*}{$\begin{array}{l}\text { Examples: foot drills/serving practice (tennis); corner } \\
\text { hit-outs/tackling drills (grass hockey); kicking/marking } \\
\text { drills (Australian Rules Football) }\end{array}$} & Session 4 (FOC only) & \multirow{2}{*}{$\begin{array}{l}\text { Dependent on sport goals and } \\
\text { surgical procedure }\end{array}$} \\
\hline & & Session 6-7 all others & \\
\hline
\end{tabular}

rehabilitation program. Participants will gradually increase their physical activity levels and return to exercise and sport based on the information brochure provided.

\section{Physiotherapy treatment integrity}

The integrity of the physiotherapy intervention will be ensured by a variety of methods. Therapist adherence to the protocol will be maximised through provision of a comprehensive treatment manual and attendance at a one-day training course outlining the study protocol and treatment program. After trial commencement, online or telephone meetings will be held to discuss any issues experienced and solutions will be instigated. Physiotherapists will use standardised, structured treatment recording forms, which will be audited by research staff. Participants will be questioned at the end of their treatment about their physiotherapy treatment experience.

\section{Descriptive data}

Age, gender, occupation, sporting involvement, duration of hip symptoms, previous treatments, medication use, imaging and surgical findings, and surgical intervention will be obtained by questionnaire, from imaging scans and from the surgical report.

\section{Outcome measures}

Outcome measures are summarised in Table 5.

\section{Patient reported outcomes}

The International Hip Outcome Tool (iHOT-33) is a recently developed self-administered tool that measures health-related quality of life in young active patients with hip disorders [23].This 33-item questionnaire covers four domains including: symptoms and functional limitations (16 items); sports and recreational activities (6 items); job related concerns (4 items); and social, emotional and lifestyle concerns (7 items). It uses a $100 \mathrm{~mm}$ horizontal visual analogue scale response format with scores ranging from 0 to 100 for each question where a higher score represents better quality of life. The overall score is calculated by taking the average out of 100 for the completed questions. The item relating to sexual activity will be omitted for ethical reasons, due to the inclusion of 16-18 year-olds in this study. For those patients who are not working for reasons other than their hip joint problems, the job-related questions are omitted and the overall score still calculated by taking the average out of 100 for the remaining questions [23]. This tool has good test retest reliability (intraclass correlation coefficient of 0.78), demonstrated face, content and construct validity and is highly responsive to clinical change (effect size of 2.0, standardised response mean of 1.7, responsiveness ratio of 6.7) [23]. In a recent comparison of the reliability of patient reported outcomes for young active adults (mean age 24 years) with FAI, we found the iHOT 33 to have a test retest reliability slightly higher than that previously estimated in an older cohort (intraclass correlation coefficient of 0.92 for total score). It is also one of the patient-reported outcomes that contain the highest number of items of particular relevance to a younger cohort, such as sports and recreation and jobrelated issues [24].

The Hip Outcome Scale (HOS) is a self-administered questionnaire designed to assess function in patients undergoing hip arthroscopy [1]. It assesses the degree of difficulty in performing tasks in two domains; activities of daily living (ADL, 17 items) and sport (9 items). The items are scored on a five point Likert scale from 4 to 0 with 4 being 'no difficulty' and 0 being 'unable to do'. The score on each of the items are summed and divided by the highest potential score (68 for the ADL subscale and 36 for the sport subscale) then multiplied by 100 to obtain a percentage. In younger patients undergoing hip arthroscopy, the HOS has excellent test re-test reliability (intraclass correlation coefficient values of 0.92 for the sports subscale and 0.98 for the ADL subscale), evidence of content, construct and concurrent validity [1,25] and is responsive to clinical change (effect sizes of 1.5 and 1.2 for the Sports and ADL subscales, respectively and area under the receiver operating characteristic curves of 0.90 and 0.88 ) [25]. Although our reliability estimates for the HOS (ICC 0.73-0.9) [24] were lower than those reported in a slightly older (mean age 33 years) cohort of patients following hip arthroscopic surgery (ICC 0.92 
Table 5 Outcome measures

\begin{tabular}{|c|c|c|}
\hline Primary outcome measures & Data collection instrument & Collection points \\
\hline Health-related quality of life & iHOT-33 & $0,14,24$ weeks \\
\hline Function in sport & Sport subscale of HOS & $0,14,24$ weeks \\
\hline \multicolumn{3}{|l|}{ Secondary outcome measures } \\
\hline $\begin{array}{l}\text { Symptoms, pain, function in daily living and sport, } \\
\text { participation in physical activities hip/groin-related quality of life }\end{array}$ & HAGOS & $0,14,24$ weeks \\
\hline Physical function & Activities of daily living subscale of HOS & $0,14,24$ weeks \\
\hline Activity level & Modified Tegner Activity Scale & $0,14,24$ weeks \\
\hline Sport participation & Heidelberg Sports Activity Scale & $0,14,24$ weeks \\
\hline Global rating of overall change & $\begin{array}{l}\text { Perceived overall change in hip/groin symptoms } \\
\text { compared to baseline }-7 \text { point ordinal scale }\end{array}$ & 14,24 weeks \\
\hline \multicolumn{3}{|l|}{ Other measures } \\
\hline Patient demographics, past treatment & Questionnaire & 0 weeks \\
\hline Surgical procedure & Post-surgery letter from surgeon to referring doctor & Following surgery \\
\hline Adverse events & Patient logbook, questionnaire & 14 weeks \\
\hline Other treatments/co-interventions & Questionnaire & 14,24 weeks \\
\hline Physiotherapy session attendance & Therapist treatment records & During intervention \\
\hline Medication use & Questionnaire & 14 weeks \\
\hline \multirow[t]{2}{*}{ Home/gym program adherence } & Participant log book - number of days/times completed & Daily during intervention \\
\hline & Self-rated using 11-point numeric rating scale & 14 weeks \\
\hline
\end{tabular}

iHOT-33= International Hip Outcome Tool.

HOS=Hip Outcome Score.

HAGOS=Copenhagen Hip and Groin Outcome Score.

† $0=$ baseline 2 weeks pre operatively; 14 and 24 weeks are timed from the date of surgery.

0.98 [24]), in two recent systematic reviews of the clinimetric properties of patient-reported outcome questionnaires to assess hip and groin disability, the HOS was recommended by both for evaluating patients undergoing hip arthroscopy [26,27].

The Copenhagen Hip and Groin Outcome Score (HAGOS) is a newly-developed patient-reported questionnaire specifically designed for young to middle-aged, physically active individuals with hip and groin pain [27]. It is a quantitative measure of the person's hip and groin disability according to the different levels of the International Classification of Functioning. The HAGOS consists of 37 items in six separate subscales relating to the past week, assessing pain (10 items), symptoms (7 items), physical function in daily living (5 items), physical function in sport and recreation (8 items), participation in physical activities (2 items) and hip and/ or groin-related quality of life (5 items). Items are scored on a five-point Likert scale of 0 to 4 , where 0 indicates no difficulty and 4 indicates extreme difficulty. A normalised score (100 indicating no symptoms and 0 indicating extreme symptoms) is calculated for each subscale. This questionnaire has been shown to have adequate measurement properties in young to middle-aged patients with chronic hip/groin pain including evidence of construct validity, responsiveness to change [27] and good to excellent test-retest reliability (intraclass correlation coefficients 0.82 to 0.91 [27] and 0.79-0.94 for the subscale [24]. Inclusion of the HAGOS will allow us to assess similarity of results from this and other selfreported measures included.

At each re-assessment time, participants will rate their perceived overall change in their hip/groin problem (compared to baseline) on a seven-point ordinal scale (much worse to much better). Scales of this kind are frequently used as an external criterion for comparison with changes in scores of other outcomes [28]. Measuring participant-perceived change using a rating of change scale has been shown to be a clinically relevant and stable method of identifying improvements that are truly meaningful from the individual perspective [29].

A modified Tegner Activity Scale and the Heidelberg Sports Activity Score will be administered to grade participants' level of physical activity (both sport/recreation and occupational) $[30,31]$.

\section{Adherence in the physiotherapy group}

The number of physiotherapy visits will be recorded for the PT group. Participants in this group will maintain a log-book to record the frequency, intensity and duration of the exercises in their home/aquatic/gym rehabilitation program. They will also rate their adherence to the 
home program overall, as well as to its separate components (gym program, pool sessions and home exercises), at 14 weeks post-surgery on 11-point numeric rating scales (with 0 being 'not at all' to 10 being 'completely as instructed').

\section{Adverse events, co-interventions and medication use}

Information on adverse events, co-interventions and medication use will be collected during the intervention phase using a log book in both groups as well as by self report questionnaire at 14 and 24 weeks post surgery. The CON group will record physical activity performed each week (type, duration and intensity), and specifically whether they consulted a physiotherapist following surgery.

\section{Sample size}

The primary endpoint will be change from baseline to 14 weeks post-surgery in (i) the iHOT-33 and (ii) sport subscale of the HOS. The minimum clinically important difference to be detected in this patient population is 6.1 for the iHOT-33 [23] and 6.0 for the Sport subscale of the HOS [24]. Between-participant standard deviations have not been widely reported in the literature for these questionnaires so the study was powered to detect a moderate effect size of 0.5 . Given this, the required sample for a two-tailed comparison of the two groups using analysis of covariance with baseline values as covariates, when $d=0.5$, power is 0.8 and type I error is 0.05 is 41 participants per group. To allow for a $15 \%$ dropout rate a total of 100 participants will be recruited.

\section{Data and statistical analysis}

A biostatistician will oversee the blinded analyses of the data. The primary analysis of the data will be undertaken using the principle of intention-to-treat. This analysis will include all participants including those who have missing data and those who do not fully adhere to the protocol. Some attrition is anticipated despite the fact that we will implement procedures to minimise loss to follow-up and participant withdrawal, and maximise adherence. To account for missing data, multiple imputation of missing follow-up measures, assuming missing data are missing at random and follow a multivariate normal distribution [32] will be performed as a sensitivity analysis.

Demographic and clinical characteristics as well as baseline data will be presented to assess the baseline comparability of the intervention groups. These variables will also be examined for those participants who withdraw from the study and those who remain.

Descriptive statistics will be presented for each group as the mean change (standard deviation, 95\% confidence intervals) in the outcomes from baseline to each time point. For continuous outcome measures, differences in mean change will be compared between groups using linear regression random effects modelling adjusted for baseline values of the outcome. Model diagnostic checks will utilise residual plots. Similar regression models for binary and ordinal outcome measures will use random effects logistic and proportional odds models, respectively. We will also perform a per protocol analysis as appropriate.

No statistical adjustment will be made for multiple testing. All tests will be two sided and carried out at the $5 \%$ level of significance. Any changes to the study design or analysis plan will be documented with full justification.

\section{Timeline}

Ethics approval from the Human Research Ethics Committee of the University of Melbourne was obtained in October 2012. Recruitment and training of the project physiotherapists occurred in February 2013. Recruitment of participants commenced in June 2013. All participants are expected to have completed the study by end December 2015.

\section{Discussion}

This paper has presented the protocol for an ongoing RCT to investigate the effect of a physiotherapysupervised rehabilitation intervention on a range of selfreported outcomes in people undergoing hip arthroscopy for management of symptomatic FAI.

The study has been designed with attention to key methodological features to minimise bias including randomisation, concealed allocation and intention-to-treat analysis. The primary outcomes are reliable and valid patient-reported measures suitable for young active individuals with FAI. The study is powered to find a moderate effect size for these outcome measures and as such smaller effects may not be detected. In addition, our study includes a longer-term follow-up. While the delivery of the physiotherapy intervention by multiple community physiotherapists risks an increase in treatment variation through the influence of therapist personality and style, it allows a more practical delivery mode that mimics clinical practice and will enhance the generalisability of the study findings.

Several authors have described rehabilitation programs following hip arthroscopy for FAI $[9,10,12,33]$ and recommend an individualised and evaluation-based program. One of the limitations of our study design is the semi-structured nature of the physiotherapy intervention, which to some extent restricts individual tailoring of the program. However, as with all controlled trials, it is optimal that treatment variation is reduced and that the intervention is accurately reported and easily replicated. Some components of the protocol used by the authors in clinical practice have been modified or 
removed for this reason: the option of dry needling has been replaced by trigger point massage only, and high velocity manipulation of the lumbar spine not included, given that not all physiotherapists have the expertise or additional qualifications required to deliver these interventions.

The findings of the study will help determine whether formal rehabilitation improves outcomes following hip arthroscopy as compared to an educational brochure, and will help guide treatment decision-making.

\section{Additional files}

\section{Additional file 1: Exercise sheets for deep hip rotator muscle strengthening.}

Additional file 2: Return to sport guidelines provided to study physiotherapists.

\section{Competing interests}

The authors declare that they have no competing interests.

\section{Authors' contributions}

AT, JOD, KLB and RSH conceived the project; KLB and JOD procured the project funding; $\mathrm{KLB}$ and $\mathrm{RSH}$ will co-ordinate the trial. KLB, RSH, AT, JOD, LS and $\mathrm{DJH}$ assisted with protocol design. AT and LS designed the physiotherapy protocol and, along with $K L B$, trained the physiotherapists. KLB wrote the first draft of this manuscript. MS performed the sample size calculations and designed the randomisation schedule and statistical analyses. All authors participated in the trial design, provided feedback on drafts of this paper and read and approved the final manuscript.

\section{Acknowledgements}

This trial is being funded by the National Health and Medical Research Council (Program Grant \#631717), St Vincent's Private Hospital East Melbourne Campus and the Australian Hip Arthroscopy Education and Research Foundation. KLB and DJH are funded in part by an Australian Research Council Future Fellowship.

The YMCA is providing the participants in the physiotherapy group with free gym and pool access during their three-month, post-surgery treatment period.

The orthopaedic surgeons identifying potential research participants are $\mathrm{Mr}$ John O'Donnell, Mr Camdon Fary, Mr Parminder Singh, Mr Phong Tran and Mr Andrew Chia.

The study physiotherapists providing the physiotherapy treatments are: Tony Beecroft, Essendon/Blackburn; Cara Peake, Ivanhoe; Michael Pierce, Ballarat; Simon Ellis, Ballarat; Craig Mansfield, Bendigo; Christian Becker, Narre Warren; Amir Takla, East Melbourne/Richmond/Ivanhoe/Blackburn; Simon Ata, Ivanhoe; Michael Rafla, Blackburn.

\section{Author details}

${ }^{1}$ The University of Melbourne, Centre for Health, Exercise and Sports Medicine, Department of Physiotherapy, School of Health Sciences, Melbourne, Vic, Australia. 'St Vincent's Private Hospital, Melbourne, Vic, Australia. ${ }^{3}$ Ivanhoe Physiotherapy Clinic, Melbourne, Vic, Australia. ${ }^{4}$ Royal North Shore Hospital, Rheumatology Department, Sydney, NSW, Australia and Kolling Institute, University of Sydney, Sydney, NSW, Australia. ${ }^{5}$ Department of Clinical Epidemiology at Cabrini, Monash University, Melbourne, Vic, Australia.

Received: 16 February 2014 Accepted: 19 February 2014 Published: 26 February 2014

\section{References}

1. Martin RL, Philippon MJ: Evidence of validity for the hip outcome score in hip arthroscopy. Arthroscopy 2007, 23(8):822-826.
2. Brunner A, Horisberger M, Herzog RF: Sports and recreation activity of patients with femoroacetabular impingement before and after arthroscopic osteoplasty. Am J Sports Med 2009, 37(5):917-922.

3. Byrd JW: Femoroacetabular impingement in athletes, part 1: cause and assessment. Sports health 2010, 2(4):321-333.

4. Anderson SE, Siebenrock KA, Tannast M: Femoroacetabular impingement. Eur J Radiol 2012, 81(12):3740-3744

5. Ganz R, Leunig M, Leunig-Ganz K, Harris WH: The etiology of osteoarthritis of the hip: an integrated mechanical concept. Clin Orthop Relat Res 2008, 466(2):264-272.

6. Agricola R, Heijboer MP, Bierma-Zeinstra SM, Verhaar JA, Weinans H, Waarsing JH: Cam impingement causes osteoarthritis of the hip: a nationwide prospective cohort study (CHECK). Ann Rheum Dis 2013, 72(6):918-923.

7. Kemp JL, Collins NJ, Makdissi M, Schache AG, Machotka Z, Crossley K: Hip arthroscopy for intra-articular pathology: a systematic review of outcomes with and without femoral osteoplasty. Br J Sports Med 2012, 46(9):632-643

8. Enseki KR, Martin RL, Draovitch P, Kelly BT, Philippon MJ, Schenker ML: The hip joint: arthroscopic procedures and postoperative rehabilitation. J Orthop Sports Phys Ther 2006, 36(7):516-525.

9. Stalzer S, Wahoff M, Scanlan M: Rehabilitation following hip arthroscopy. Clin Sports Med 2006, 25(2):337-357.

10. Philippon MJ, Christensen JC, Wahoff MS: Rehabilitation after arthroscopic repair of intra-articular disorders of the hip in a professional football athlete. J Sport Rehabil 2009, 18(1):118-134.

11. Enseki KR, Martin R, Kelly BT: Rehabilitation after arthroscopic decompression for femoroacetabular impingement. Clin Sports Med 2010, 29(2):247-255. viii.

12. Edelstein J, Ranawat A, Enseki KR, Yun RJ, Draovitch P: Post-operative guidelines following hip arthroscopy. Curr Rev Musculoskelet Med 2012, 5(1):15-23.

13. Jayasekera N, Aprato A, Villar RN: Are crutches required after hip arthroscopy? A case-control study. Hip Int 2013, 23(3):269-273.

14. Pierce CM, Laprade RF, Wahoff M, O'Brien L, Philippon MJ: Ice hockey goaltender rehabilitation, including on-ice progression, after arthroscopic hip surgery for femoroacetabular impingement. J Orthop Sports Phys Ther 2013, 43(3):129-141.

15. Boutron I, Moher D, Altman DG, Schulz KF, Ravaud P: Extending the CONSORT statement to randomized trials of nonpharmacologic treatment: explanation and elaboration. Ann Intern Med 2008, 148(4):295-309.

16. Tönnis D: Congenital Dysplasia and Dislocation of the Hip in Children and Adults. Berlin: Springer-Verlag; 1987. English Edition.

17. Sluka K: Mechanisms and Management of Pain for the Physical Therapist. Seattle: IASP Press; 2009.

18. Maitland GD: Maitland's Vertebral Manipulation. 6th edition. Oxford; Boston: Butterworth-Heinemann; 2001.

19. Retchford TH, Crossley KM, Grimaldi A, Kemp JL, Cowan SM: Can local muscles augment stability in the hip? A narrative literature review. J Musculoskelet Neuronal Interact 2013, 13(1):1-12.

20. Torry MR, Schenker ML, Martin HD, Hogoboom D, Philippon MJ: Neuromuscular hip biomechanics and pathology in the athlete. Clin Sports Med 2006, 25(2):179-197. vii.

21. Decker M: Deep hip muscle activiation during a squat exercise. In Conference Proceedings of the Annual Meeting of the American Society of Biomechanics: 2009: 2009:1

22. Giphart JE, Stull JD, Laprade RF, Wahoff MS, Philippon MJ: Recruitment and activity of the pectineus and piriformis muscles during hip rehabilitation exercises: an electromyography study. Am J Sports Med 2012, 40(7):1654-1663.

23. Mohtadi NG, Griffin DR, Pedersen ME, Chan D, Safran MR, Parsons N, Sekiya JK, Kelly BT, Werle JR, Leunig M, McCarthy JC, Martin HD, Byrd JWT, Philippon MJ, Martin RL, Guanche CA, Clohisy JC, Sampson TG, Kocher MS, Larson CM: The development and validation of a self-administered quality-of-life outcome measure for young, active patients with symptomatic hip disease: the International Hip Outcome Tool (iHOT-33). Arthroscopy 2012, 28(5):595-605. quiz 606-510 e591.

24. Hinman RS, Dobson F, Takla A, O'Donnell J, Bennell KL: Which is the most useful patient-reported outcome in femoroacetabular impingement? Test-retest reliability of six questionnaires. Br J Sports Med 2013. doi:10.1136/bjsports-2012-092072, in press. 
25. Martin RL, Philippon MJ: Evidence of reliability and responsiveness for the hip outcome score. Arthroscopy 2008, 24(6):676-682

26. Lodhia P, Slobogean GP, Noonan VK, Gilbart MK: Patient-reported outcome instruments for femoroacetabular impingement and hip labral pathology: a systematic review of the clinimetric evidence. Arthroscopy 2011, 27(2):279-286.

27. Thorborg K, Holmich P, Christensen R, Petersen J, Roos EM: The Copenhagen Hip and Groin Outcome Score (HAGOS): development and validation according to the COSMIN checklist. Br J Sports Med 2011, 45(6):478-491.

28. Jaeschke R, Singer J, Guyatt GH: Measurement of health status. Ascertaining the minimal clinically important difference. Control Clin Trials 1989, 10(4):407-415.

29. ten Klooster PM, Drossaers-Bakker KW, Taal E, van de Laar MA: Patientperceived satisfactory improvement (PPSI): interpreting meaningful change in pain from the patient's perspective. Pain 2006, 121(1-2):151-157.

30. Tegner $Y$, Lysholm J: Rating systems in the evaluation of knee ligament injuries. Clin Orthop Relat Res 1985, 198:43-49.

31. Seeger J, Weinmann S, Schmitt H, Bruckner T, Krueger M, Clarius M: The Heidelberg sports activity score - a new instrument to evaluate sports activity. Open Orthop J 2013, 7:25-32.

32. Molenberghs GaK M: Missing Data in Clinical Studies. Chichester: Wiley; 2007.

33. Wahoff M, Ryan M: Rehabilitation after hip femoroacetabular impingement arthroscopy. Clin Sports Med 2011, 30(2):463-482

doi:10.1186/1471-2474-15-58

Cite this article as: Bennell et al:: Efficacy of a physiotherapy rehabilitation program for individuals undergoing arthroscopic management of femoroacetabular impingement - the FAIR trial: a randomised controlled trial protocol. BMC Musculoskeletal Disorders 2014 15:58.

\section{Submit your next manuscript to BioMed Central and take full advantage of:}

- Convenient online submission

- Thorough peer review

- No space constraints or color figure charges

- Immediate publication on acceptance

- Inclusion in PubMed, CAS, Scopus and Google Scholar

- Research which is freely available for redistribution 